\title{
Is genetic screening for the Lynch syndrome effective?
}

Hampel H, Frankel WL, Martin E, Arnold M, Khanduja K, Kuebler $\mathrm{P}$, et al. Screening for the Lynch syndrome (hereditary nonpolyposis colorectal cancer). N Engl J Med 2005;352:1851-60.

Background: Probably about $3 \%-4 \%$ of cases of colorectal cancer can be attributed to a hereditary predisposition, such as familial adenomatous polyposis and the Lynch syndrome (hereditary nonpolyposis colorectal cancer). ${ }^{1}$ Colorectal tumours in people with the Lynch syndrome are usually proximal to the splenic flexure, are often multiple and are associated with endometrial and breast cancer. ${ }^{2}$ The age of onset is about 45 years. Until recently, patients with the Lynch syndrome were identified by these phenotypic features according to clinical diagnostic criteria such as the Amsterdam II criteria and the Bethesda guidelines. However, with the discovery in the early 1990s that germ-line mutations in the DNA mismatch-repair genes MSH2, MLH1, MSH6 and PMS2 lead to the development of the Lynch syndrome, it has become possible to perform genetic screening to identify patients with this syndrome. What proportion of people with colorectal cancer have the Lynch syndrome, and which method of screening for mismatch-repair deficiency is more effective?

Design: The authors enrolled 1581 patients with newly diagnosed colorectal adenocarcinoma at 6 hospitals serving metropolitan Columbus, Ohio, between 1999 and 2004. Family history, blood samples for DNA and RNA extraction, and tissue specimens were obtained from 1066 patients who had undergone genotyping of the tumour for microsatellite instability (a marker of the genetic abnormality characteristic of the Lynch syndrome) at the time of data analysis. Immunohistochemical analysis, another method of screening for mismatch-repair deficiency, was performed in 208 patients positive for microsatel- lite instability and in 109 patients with no microsatellite instability but who were at high risk of cancer according to specified clinical criteria. To identify which patients with microsatellite instability had the Lynch syndrome, methylation analysis of the MHL1 promoter region was performed (cancers with microsatellite instability that show hypermethylation in the promoter region are not considered to be due to the Lynch syndrome). Confirmation of the syndrome was done by mutation analysis. Probands with deleterious mutations were offered genetic counselling. The relatives of patients with confirmed Lynch syndrome were offered genetic counselling and mutation testing.

Results: A deleterious mutation in a mismatch-repair gene was found in $23(2.2 \%)$ of the 1066 patients with colorectal cancer, none of whom had previously received a diagnosis of the Lynch syndrome. The mean age of these patients at diagnosis of colorectal cancer was 50.4 years (range 23-87). Only 3 fulfilled the Amsterdam criteria; an additional 15 met the Bethesda criteria. Genetic counselling was received by 21 of the 23 probands and 117 of their relatives. Molecular testing of the relatives revealed that 52 were positive for the mutation and 14 of them had had a cancer related to the Lynch syndrome. The effectiveness of the 2 screening techniques - genotyping for microsatellite instability and immunohistochemical analysis was comparable in cases with high-frequency microsatellite instability (instability present in 2 or more markers) but not in those with low-frequency microsatellite instability.

Commentary: This study pro- vides additional evidence of the usefulness of molecular testing for genetic susceptibility to cancer. The Amsterdam criteria and the Bethesda guidelines missed a substantial number of cases of the Lynch syndrome detected with the use of microsatellite instability testing and immunohistochemical analysis. However, these 2 tests cannot be recommended as screening methods in all patients with colorectal cancer, since they have low yield among patients over 55 years old, ${ }^{1}$ are time-consuming and require expert interpretation. The cost-effectiveness of such screening has yet to be determined, and the screening criteria should be tested in a larger population.

Practice implications: The results of this study show that microsatellite instability testing and immunohistochemical analysis performed in carefully selected patients could be useful in identifying those with the Lynch syndrome. Such identification is important because patients with the syndrome have a different prognosis (usually better) than other patients with colorectal cancer, their tumours respond differently to chemotherapy (those with high-frequency microsatellite instability do not respond to fluorouracil-based adjuvant chemotherapy), and they often have other cancers. Relatives of patients with the Lynch syndrome should be offered early testing and regular checkups, not only to detect colorectal cancer but also to detect other tumours that often accompany the Lynch syndrome.

Aleksandra Mišak

Editorial Fellow, CMAJ

\section{References}

1. Lynch HT, Lynch PM. Molecular screening for the Lynch syndrome Better than family history? [editorial] NEngl 7 Med 2005;352:1920-2.

2. Lynch HT, Lynch J. Lynch syndrome: genetics, natural history, genetic counseling, and prevention. I Clin Oncol 2000;18(21 Suppl):19S-31S. 